\title{
La succession végétale sur les cultures en terrasses de la Vallée de la Roya (Alpes-Maritimes, France) et sa perception par la population locale
}

\author{
Sébastien Boillat, Conradin A. Burga, Andreas Gigon, \\ Norman Backhaus, Zürich
}

\section{Les terrasses de culture: un patrimoine à l'abandon}

Là où les Alpes rejoignent la Méditerranée, le relief escarpé a contraint les cultivateurs à aménager les versants en terrasses, au prix d'un travail colossal qui s'étale souvent sur plusieurs siècles (BLANCHEMANCHE 1990). Les terrasses, destinées à l'origine à la culture céréalière et maraîchère, offraient une protection optimale contre l'érosion du sol, une plus grande profondeur de sol et une meilleure infiltration de l'eau, souvent complétée par un réseau d'irrigation (FRAPA et al. 1989).

Dans la région, la déprise agricole s'est effectuée en deux phases. Au début du 20ème siècle, les cultures céréalières et maraîchères ont été remplacées par l'élevage sur prairies sèches (BoTTON 1996), qui a, à son tour, disparu progressivement depuis 1945. Actuellement, la majeure partie des terrasses est à l'abandon, car elles ne permettent pas l'emploi de grandes machines agricoles et demandent beaucoup d'entretien. Les murs s'écroulent et les terrains sont laissés en friche, avec des conséquences écologiques et culturelles: la progression des friches sur les prairies sèches provoque une diminution de la diversité et du nombre d'espèces (KöHLER 2001); dans le cas des terrasses, la perte irréversible du patrimoine paysager et du savoir-faire agricole séculaire y est liée.

Dans la vallée de la Roya (Alpes-Maritimes), où presque toutes les terres cultivables ont été aménagées en terrasses, la déprise agricole a été très forte et la majorité des terres est à l'abandon. Cependant, depuis une trentaine d'années, dans la mouvance des idéologies nées autour de mai 1968, des néo-ruraux, venus des grandes villes de France et parfois d'Allemagne et de Suisse, se sont installés dans la vallée, avec l'idéal d'y vivre de la terre et d'inverser ce processus. Finalement, la vallée de la Roya est caractérisée par une exceptionnelle diversité paysagère et floristique inhérente à sa situation géographique (OzENDA 1981).

\section{Buts et zone de l'étude}

Face aux problèmes économiques et démographiques de l'abandon des terrasses, on peut se demander quel est l'avenir d'une zone rurale dans laquelle l'olivier a été surtout cultivé, comment l'environnement peut y évoluer et comment la population locale perçoit ces changements.

La présente étude est divisée en deux parties. La première, écologique, a pour but de comprendre le processus de succession végétale en décrivant l'état actuel de la végétation sur les anciennes cultures en terrasses et les facteurs qui l'influencent. Comme les points de départ de la succession sont aussi des oliveraies, nous parlons de succession sur les cultures en non pas seulement d'une succession sur des prairies.

La seconde partie de l'étude, socioculturelle, a pour but d'évaluer qualitativement l'opinion de la population locale à propos de l'abandon des terres, ainsi que ses visions d'avenir pour la vallée.

En regroupant ces résultats, l'objectif est d'élaborer des scénarios sur l'évolution possible du paysage dans la vallée.

La zone d'étude comprend toutes les zones aménagées en terrasses (oliviers, arbres fruitiers) situées entre 300 et $700 \mathrm{~m}$ d'altitude sur le territoire des trois communes de Breil-sur-Roya, Saorge et Fontan.

\section{Méthodes}

\subsection{Méthodes écologiques}

Quarante-cinq relevés, selon la méthode de BraUnBlanquet (1964), ont permis d'identifier et de décrire 14 types de végétation. En comparant les différents types de végétation, un modèle de succession a pu être élaboré selon le principe de «space-for-time substitution» (GLAVAC 1996). Les types de végétation ont été cartographiés et le pourcentage de la surface des types de végétation a été calculé pour 7 différents secteurs de la zone d'étude au moyen du logiciel MapFactory®. L'état des terrasses a été estimé sur une échelle de 1 à 5 (tableau 1) pour chaque station faisant l'objet d'un relevé, et mis en relation avec l'état de la végétation et de la pente.

\subsection{Méthodes socioculturelles}

En suivant un questionnaire standard décrit dans Boillat (2002), 21 interviews ont été réparties comme l'indique le tableau 2.

D'après une enquête préliminaire (LAMNEK 1995), les 


\begin{tabular}{|l|l|l|}
\hline Degré & État & Description \\
\hline 1 & $<10 \%$ écroulé & presque in tact \\
\hline 2 & $10-33 \%$ écroulé & environ 1 tiers écroulé \\
\hline 3 & $>33-67 \%$ écroulé & environ la moitié écroulée \\
\hline 4 & $>67-90 \%$ écroulé & environ 2 tiers écroulés \\
\hline 5 & $>90 \%$ écroulé & détruit \\
\hline
\end{tabular}

Tab. 1: Echelle d'évaluation de l'état des terrasses Scale for the assessment of the state of the terraces Skala für die Beurteilung des Zustandes der Terrassen néo-ruraux pourraient avoir une opinion différente des autres villageois par rapport à l'abandon des terrasses. Pour vérifier cette hypothèse, les catégories de personnes ont été définies par rapport aux paramètres qui définissent la néo-ruralité: l'origine (originaire ou non de la vallée) et la profession (agriculteur ou non). Ces catégories sont donc: (1) les néo-ruraux, venus dans la vallée dans le but d'y vivre de l'agriculture, mais qui exercent parfois des activités annexes, (2) les agriculteurs originaires de la vallée, (3) les personnes non originaires de la vallée venues s'y installer sans être agriculteurs, et (4) les non-agriculteurs originaires de la vallée.

Il faut remarquer que le nombre peu élevé d'interviews ne permet pas une étude représentative et quantitative de la population de la vallée par rapport au problème des terrasses. Mais les personnes interviewées peuvent être considérées comme caractéristiques, dans la mesure où (1) une diversité maximale de types de personnes (selon l'âge, la profession et l'origine) ont été interrogées, et (2) le critère de redondance ou saturation théorique (FLICK 2000) a été atteint dans le sens où, à l'intérieur d'un même groupe, de nouvelles opinions cessent d'apparaître après avoir interrogé un certain nombre de personnes.

\section{Les terrasses: présent, succession et répartition dans l'espace}

\subsection{Les terrasses encore entretenues: oliveraies et prairies sèches}

À Breil, la majeure partie des terrasses encore entretenues sont des oliveraies (fig.1). Ce sont des plantations parfois anciennes mais toujours productives. Il n'existe qu'une seule exploitation à but commercial, fondée il y a quelques années par un jeune couple. Sous les oliviers, on trouve une prairie fauchée au moins chaque année à Arrhenatherum elatius, qui signale une fumure modérée (ELLENBERG 1996).

A Saorge, on trouve une extension importante de terrasses exploitées en prairies sèches à Bromus erectus, régulièrement fauchées, en majorité par des néo-ruraux pour l'élevage de gros bétail, bien que

\begin{tabular}{|l|l|}
\hline Age & $\begin{array}{l}\text { Nombre } \\
\text { personnes }\end{array}$ \\
\hline$<35$ & 7 \\
\hline $\begin{array}{l}35- \\
65\end{array}$ & 9 \\
\hline$>65$ & 5 \\
\hline
\end{tabular}

\begin{tabular}{|l|l|}
\hline Village & $\begin{array}{l}\text { Nombre } \\
\text { personnes }\end{array}$ \\
\hline Breil & 12 \\
\hline Saorge & 7 \\
\hline Fontan & 1 \\
\hline Tende & 1 \\
\hline
\end{tabular}

\begin{tabular}{|l|c|}
\hline Sexe & $\begin{array}{l}\text { Nombre } \\
\text { personnes }\end{array}$ \\
\hline Femmes & 5 \\
\hline Hommes & 11 \\
\hline Couples* & 5 \\
\hline
\end{tabular}

\begin{tabular}{|l|l|}
\hline Catégorie & $\begin{array}{l}\text { Nb. } \\
\text { pers. }\end{array}$ \\
\hline Néo-ruraux & 7 \\
\hline Non-agriculteurs, non-originaires & 4 \\
\hline Agriculteurs originaires & 3 \\
\hline Originaires, non-agriculteurs & 7 \\
\hline
\end{tabular}

\footnotetext{
*) Des couples ont parfois donné une interview ensemble et ont été considérés comme une seule personne si leurs opinions ne montraient pas de divergences importantes.
}

Tab. 2: Répartition des 21 personnes interviewées

Typology of the 21 interview partners

Typologie der 21 Interviewpartner 


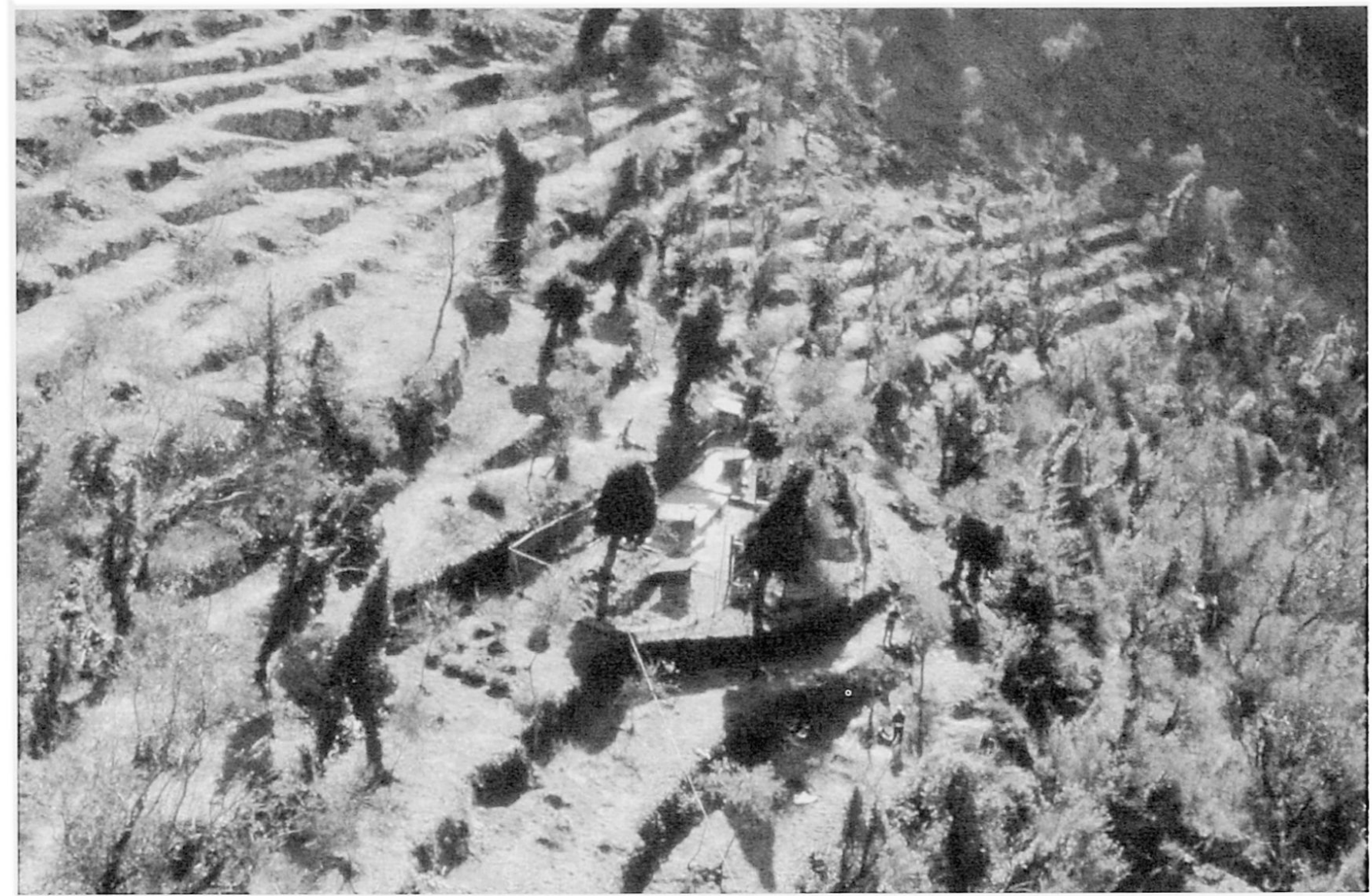

Fig. 1: Oliveraie sur terrasses entretenue près de Breil-sur-Roya Traditionally cultivated terraced olive grove near Breil-sur-Roya

Photo: C.A. Burga Traditionell genutzter terrassierter Olivenhain bei Breil-sur-Roya

ces terrasses aient été à l'origine destinées à la culture céréalière et maraîchère (FRAPA et al. 1989). L'élevage n'est devenu important dans la vallée qu'à partir du début du 20ème siècle (Botron 1996); il a l'inconvénient de contribuer à l'écroulement des murs (FrAPA et al. 1989). Néanmoins, les prairies à Bromus erectus sont très riches en espèces, entre 30 et 40 espèces pour $50 \mathrm{~m}^{2}$, comme l'ont montré les relevés d'Albrecht (2001) et Boillat (2002). C'est le cas aussi pour les orchidées, dont les collaborateurs du Parc National du Mercantour ont dénombré 45 espèces, rien que pour la vallée de la Bendola, aux alentours de Saorge (Cevasco 2001). Cette diversité exceptionnelle est menacée autant par l'éventuel apport d'engrais que par l'abandon en friche (KÖHLER 2001).

\begin{tabular}{|l|l|}
\hline Stade & Définition \\
\hline Prairie de fauche (Stade de départ) & Fauche au moins une fois par année \\
\hline Prairie en friche & Brachypodium pinnatum $>25 \%$ de couverture \\
\hline Brousse arbustive, maquis ou garrigue & Couverture arbustive $(0,4-4 \mathrm{~m})>30 \%$ \\
\hline Forêt & Couverture arborée $(>4 \mathrm{~m})>20 \%$ \\
\hline
\end{tabular}

Tab. 3: Les quatre stades de la succession à partir des prairies sur terrasses (d'après ALBRECHT 2001) The four succession stages of the grasslands on the terraces (according to ALBRECHT 2001)

Die vier Sukzessionsstadien der Wiesen auf den Terrassen (nach AlbRecht 2001) 
4.2 Les terrasses abandonnées: de la prairie à la forêt A partir des prairies décrites ci-dessus, nous pouvons distinguer quatre principaux stades de succession définis dans le tableau 3. Chacun des stades comprend plusieurs types de végétation décrits dans le tableau 4. La fig. 3 montre le déroulement probable de la succession pour ces types de végétation.

Dès l'arrêt de la fauche, les prairies à Bromus erectus et Arrhenatherum elatius se développent à moyen terme en prairie à Brachypodium pinnatum (fig. 3). Cette espèce peut devenir très dominante (RICHTER 1989) et former une couverture dense qui entrave l'établisse- ment des ligneux et ralentit le processus de succession (SchmidT 1985), à moins que des facteurs dérangeants ne viennent créer des niches où les ligneux peuvent s'établir (LEUTERT 1983). Dans la zone étudiée, l'écroulement des murs et le piétinement par les sangliers créent ces niches, si bien que peu de prairies à couverture dense de $B$. pinnatum ont été observées. Dans les oliveraies en friche, sous lesquelles se trouve une prairie à $B$. pinnatum, une proportion relativement forte de ligneux a été observée, due probablement à la fois au microclimat aux extrêmes atténués par la présence des oliviers (Richter 1989), et à l'habitat que ceux-ci offrent aux oiseaux disséminateurs des graines

\begin{tabular}{|c|c|c|}
\hline Type de végétation & Espèces dominantes & Autres espèces typiques (espèces différentielles) \\
\hline \multicolumn{3}{|l|}{ Prairies de fauche } \\
\hline Prairie sous oliveraie & Arrhenatherum elatius & $\begin{array}{l}\text { Vicia sativa, Bromus sterilis, Dactylis glomerata, } \\
\text { Trifolium campestre }\end{array}$ \\
\hline Prairie sèche & Bromus erectus & $\begin{array}{l}\text { Onobrychis viciifolia, Artemisia alba, Globularia } \\
\text { vulgaris, Hippocrepis comosa }\end{array}$ \\
\hline Prairies en friche & & 2 \\
\hline Prairie en friche sous oliveraie & Brachypodium pinnatum & $\begin{array}{l}\text { Rubus fruticosus s. l., Lathyrus sylvestris, Vicia } \\
\text { cracca ssp. incana }\end{array}$ \\
\hline Prairie en friche & Brachypodium pinnatum & $\begin{array}{l}\text { Couverture et vitalité des espèces différentielles } \\
\text { réduites par rapport aux prairies de fauche }\end{array}$ \\
\hline $\begin{array}{l}\text { Prairie en friche avec zones de } \\
\text { garrigue }\end{array}$ & $\begin{array}{l}\text { Brachypodium pinnatum, } \\
\text { Thymus vulgaris }\end{array}$ & Diplachne serotina, Globularia vulgaris \\
\hline \multicolumn{3}{|l|}{ Stade arbustif } \\
\hline Brousse caducifoliée à sumac & $\begin{array}{l}\text { Cotinus coggygria, Pistacia } \\
\text { terebinthus, Spartium junceum }\end{array}$ & Osyris alba, Saponaria ocymoides \\
\hline Brousse caducifoliée à cornouiller & $\begin{array}{l}\text { Cornus sanguinea, Prunus } \\
\text { spinosa, P. mahaleb }\end{array}$ & Verbascum chaixii, Vicia cracca \\
\hline Brousse à genêt & Spartium junceum & Ferula communis \\
\hline Maquis sempervirent & $\begin{array}{l}\text { Rhamnus alaternus, Phillyrea } \\
\text { latifolia }\end{array}$ & Peucedanum officinale, Ruta chalepensis \\
\hline Garrigue à thym & $\begin{array}{l}\text { Thymus vulgaris, Juniperus } \\
\text { phoenicea }\end{array}$ & Linum tenuifolium, Diplachne serotina \\
\hline Garrigue à ciste & $\begin{array}{l}\text { Cistus albidus, Calycotome } \\
\text { spinosa }\end{array}$ & $\begin{array}{l}\text { Aphyllantes monspeliensis, Astragalus } \\
\text { monspessulanus, Coronilla minima }\end{array}$ \\
\hline \multicolumn{3}{|l|}{ Stade forestier } \\
\hline Forêt de chênes & Quercus pubescens & $\begin{array}{l}\text { Cistus salviifolius, Sorbus domestica, Clematis } \\
\text { vitalba, Linum narbonense }\end{array}$ \\
\hline Forêt de charmes & Ostrya carpinifolia & $\begin{array}{l}\text { Acer opalus, Cornus sanguinea, Daphne laureola, } \\
\text { Melica uniflora, Euphorbia dulcis }\end{array}$ \\
\hline Forêt de pins d'Alep & Pinus halepensis & $\begin{array}{l}\text { Quercus ilex, Lonicera implexa, Pistacia lentiscus, } \\
\text { Arbutus unedo }\end{array}$ \\
\hline Forêt de pins sylvestres & Pinus sylvestris & (non étudiées) \\
\hline
\end{tabular}

Tab. 4: Types de végétation dans la région d'étude

Vegetation types of the study region

Vegetationstypen des Untersuchungsgebietes 


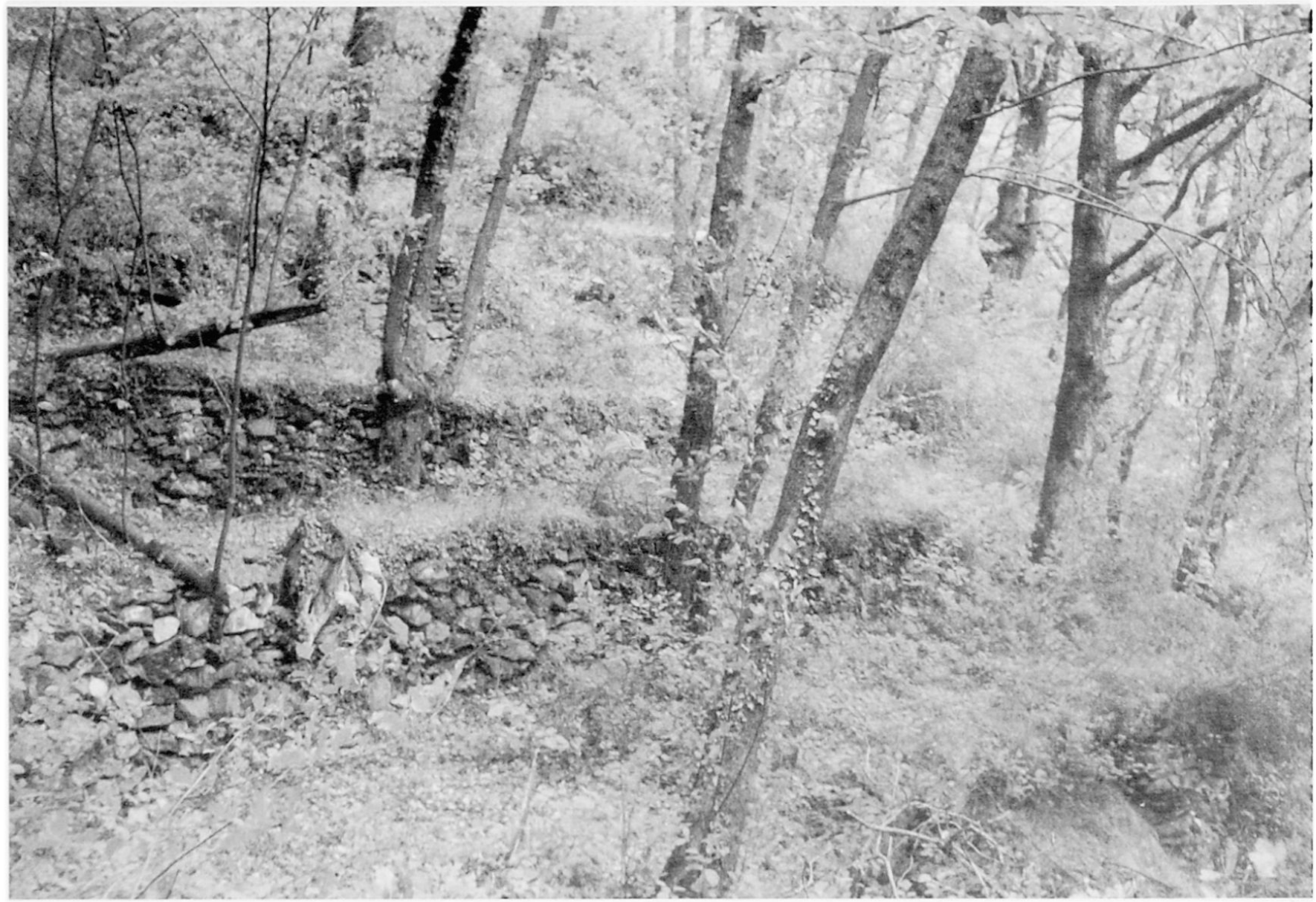

Fig. 2: Anciennes terrasses recouvertes par la forêt à Saorge Old terraces near Saorge, covered with deciduous forest Alte, von Laubwald bestockte Terrassen bei Saorge

(NE'EMAN \& IzHAK1 1996). Le stade arbustif apparaît donc probablement plus rapidement sous les oliveraies à l'abandon que sur les prairies sèches.

Le stade arbustif peut prendre des formes très diverses (tableau 4), qui sont en général déterminées par des facteurs mésoclimatiques (exposition et altitude) et édaphiques (profondeur, humidité et richesse du sol). Les zones les plus chaudes (versants sud, altitude $<400 \mathrm{~m}$ ) sont colonisées par un maquis sempervirent à Rhamnus alaternus et Phillyrea latifolia, les zones moyennement chaudes (versants est, ouest et sud audessus de $400 \mathrm{~m}$ ) par une brousse caducifoliée à Cotinus coggyria et Pistacia terebinthus, et les zones les plus fraîches (versants nord) par une brousse caducifoliée à Cornus sanguinea et Prunus spinosa. Les zones au sol plus pauvre et rocheux, sur les hauteurs et les crêtes, sont couvertes par une garrigue à Thymus vulgaris et Juniperus phoenicea, à Cistus albidus et Calycotome spinosa, ou par une brousse à Spartium junceum.
La vitesse du développement de la forêt à partir du stade arbustif dépend de l'apport en semences depuis les environs (Tatoni \& Roche 1994). Dans la vallée de la Roya, il existe des extensions importantes de forêt, ce qui favorise la propagation des essences. Partout, la présence de jeunes arbres dans les stades arbustifs nous indique leur probable évolution vers la forêt, sauf dans la brousse à Spartium junceum où les rameaux difficilement décomposables de cet arbuste s'accumulent sur le sol et peuvent entraver la germination des arbres (Richter 1989). Les forêts peuvent aussi se développer directement sur les prairies en friche, surtout si celles-ci se trouvent en lisière de forêt. Mais comme cette évolution semble plutôt exceptionnelle (peu de jeunes arbres observés dans les prairies), elle n'a pas été représentée dans la fig. 3.

Le type de forêt dépend du mésoclimat. On a des forêts de Quercus pubescens sur les versants sud (fig. 2), de Ostrya carpinifolia sur les versants nord, de Pinus hale pensis dans la partie la plus basse et de Pinus sylvestris 


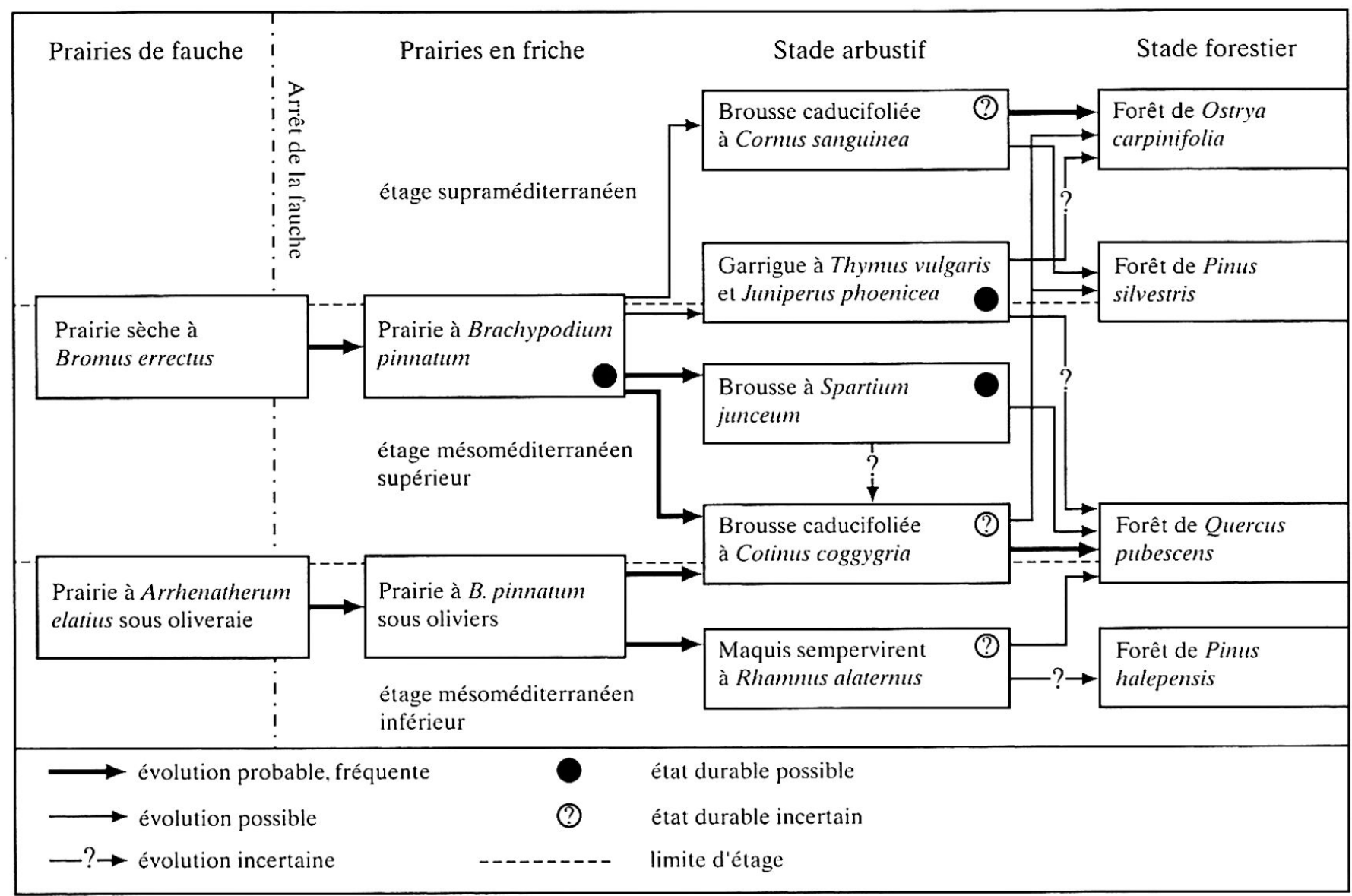

Fig. 3: Modèle de succession sur les prairies abandonnées Succession model of abandoned grassland Sukzessions-Modell zu ungenutztem Grünland

sur les hauteurs. Ces forêts, dans leurs caractéristiques et leur répartition, correspondent bien à la végétation naturelle potentielle de la région définie par OzENDA (1981) et BARBERo et al. (1973), même si ce sont encore de jeunes forêts définies par des caractères secondaires.

Nous pouvons conclure que le stade de départ de la succession (oliveraies ou prairies sèches) joue un rôle jusqu'à l'arrivée du stade arbustif (fig. 3). Ensuite, ce sont des facteurs mésoclimatiques et édaphiques qui déterminent le type de végétation. Il n'a pas été possible de décrire les premiers stades de la succession sur d'anciennes terres labourées. Celle-ci y est nettement plus rapide du fait de l'absence d'une dense couverture de graminées (ScrimidT 1993) et a déjà partout atteint le stade arbustif. Il n'est donc plus possible de distinguer si un stade arbustif était à l'origine un champ labouré ou une prairie.

\subsection{Végétation et état des terrasses}

L'état des murs des terrasses, selon l'échelle du tableau 1, n'est pas toujours en corrélation avec le stade de la succession. Il existe des terrasses fauchées régulièrement dont le propriétaire a renoncé à l'entretien des murs, trop laborieux, exigeant en outre un savoir-faire bien spécifique. La fauche des parcelles ne va donc pas de pair avec l'entretien des murs. Parfois, les murs sont encore plus endommagés par l'action du petit bétail. Par contre, certaines zones de broussailles présentent des murs en relativement bon état (degré 2 selon l'échelle) sans qu'il soit possible de savoir pourquoi. Les racines des ligneux endommagent en général les murs, mais le lierre (Hedera helix) peut aussi les protéger. L'état des murs dépend aussi de leur solidité initiale (qualité de construction, drainage, type de pierre) et d'autres facteurs comme le passage de gibier, la présence de rigoles de ruissellement après de fortes précipitations, le type de sol ou l'action du feu.

\subsection{Entretien ou abandon? Répartition dans l'espace}

La fig. 4 montre la proportion de surface de terrasses de chaque stade de la succession pour chacun des secteurs de la région étudiée. Au total, environ 30\% des terrasses sont encore fauchées. La forêt recouvre 


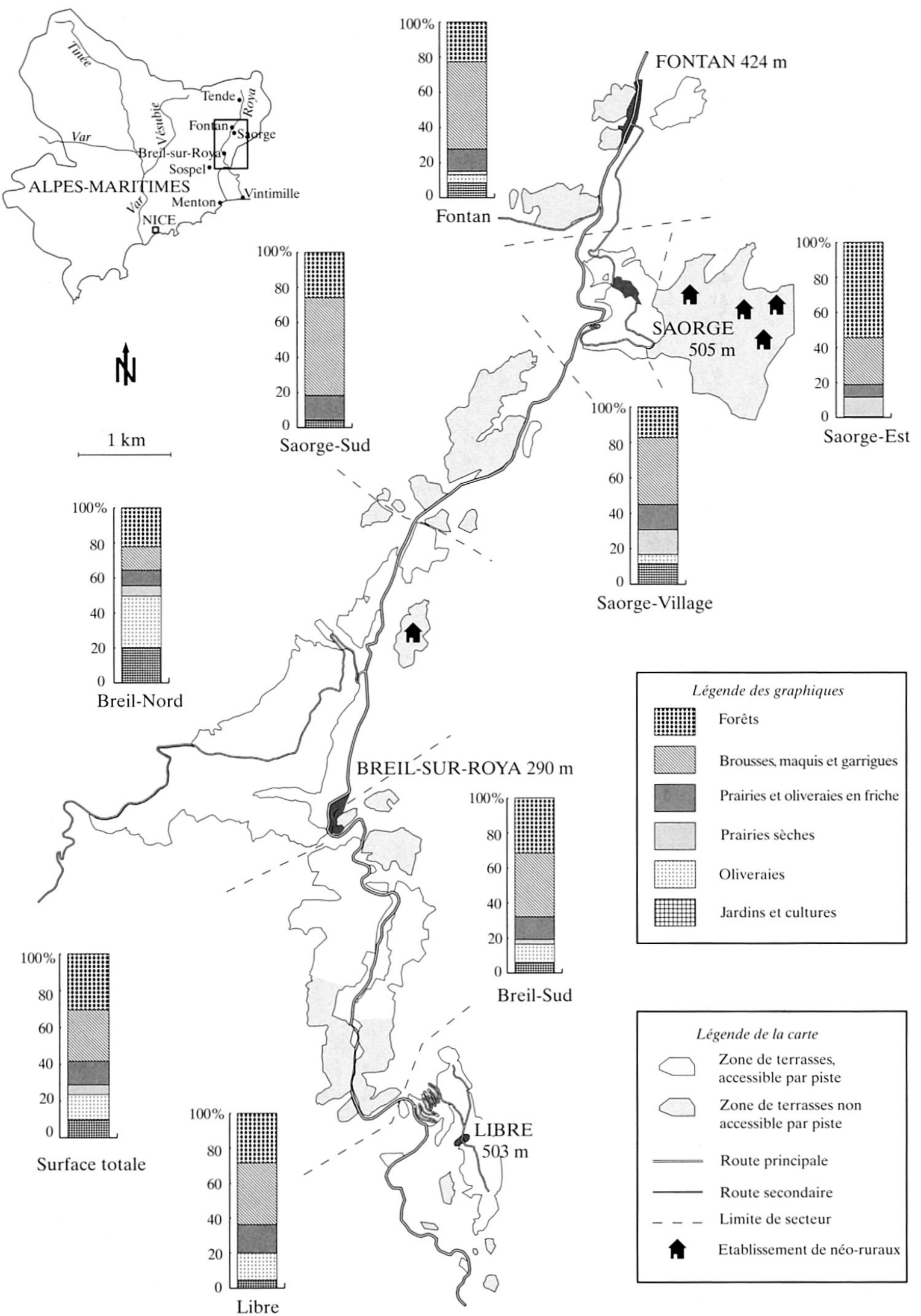

Fig. 4: Répartition géographique des stades de la succession à l'intérieur du périmètre d'investigation Range of succession stages within the investigation area Geographische Verbreitung der Sukzessions-Stadien 


\begin{tabular}{|l|l|l|l|l|l|}
\hline Eléments du paysage & $\begin{array}{l}\text { Néo- } \\
\text { ruraux }\end{array}$ & $\begin{array}{l}\text { Non-agriculteurs, } \\
\text { non-originaires }\end{array}$ & $\begin{array}{l}\text { Non-agriculteurs } \\
\text { originaires }\end{array}$ & $\begin{array}{l}\text { Agriculteurs } \\
\text { originaires }\end{array}$ & Total \\
\hline Olives & 1 & 0 & 2 & 1 & 4 \\
\hline Montagne & 2 & 0 & 2 & 0 & 4 \\
\hline Climat, soleil & 0 & 1 & 0 & 1 & 2 \\
\hline Variété paysage & 1 & 3 & 4 & 0 & 8 \\
\hline Aspect sauvage & 4 & 0 & 0 & 0 & 4 \\
\hline
\end{tabular}

Tab. 5: Eléments du paysage cités comme attractifs.

Landscape elements which were denoted as attractive.

Landschaftselemente, die als attraktiv bezeichnet wurden.

déjà $30 \%$ de la surface des terrasses, et les $40 \%$ restants sont des prairies et broussailles en friche qui, comme nous l'avons vu, évoluent à long terme vers la forêt.

La plus forte proportion de terrasses fauchées se trouve près de Breil. Il s'agit d'une vaste oliveraie située le long de la route du col de Brouis, facilement accessible. On y trouve également beaucoup de résidences secondaires qui sont souvent entourées de jardins d'ornement, parfois plantés d'espèces exotiques. Breil est le village le plus peuplé des trois avec 2069 habitants (recensement de 1999), ce qui encourage l'entretien de toute cette zone.

En revanche, les secteurs de Fontan et de Saorge, ainsi que d'une partie de Breil-Sud, ont une plus forte proportion de terrasses à l'abandon. C'est en particulier le cas pour les zones non accessibles par des pistes, comme par exemple Saorge-Sud, et aussi pour les zones à forte pente où les terrasses sont hautes et étroites et demandent plus d'entretien.
Mais le manque d'accès n'encourage pas toujours l'abandon. Dans le secteur de Saorge-Est et près du village de Saorge, on trouve une surface importante de prairies sèches de fauche non accessibles par une piste. Ces prairies sont entretenues presque exclusivement par des néo-ruraux. Plus en aval, elles sont rares à cause de l'apport d'engrais lié aux oliviers qui donne des prairies à Arrhenatherum elatius. Le maintien des prairies sèches est souvent lié à la présence des néoruraux qui les entretiennent. Nous pouvons donc conclure que les zones les plus fréquemment à l'abandon sont les plus en pente et les plus inaccessibles, excepté là où se sont installés des néo-ruraux.

\section{L'abandon des terrasses vu par la population locale}

Dans les éléments du paysage de la vallée cités comme attrayants (tableau 5), on trouve des caractéristiques du paysage méditerranéen (olives, climat, soleil) et alpin (montagne), et surtout la variété du paysage typique des Alpes-Maritimes, citée explicitement par 8 des

\begin{tabular}{|c|c|c|c|c|c|}
\hline $\begin{array}{l}\text { Mesures proposées pour } \\
\text { l'entretien des terrasses }\end{array}$ & $\begin{array}{l}\text { Néo- } \\
\text { ruraux }\end{array}$ & $\begin{array}{l}\text { Non-agriculteurs, } \\
\text { non-originaires }\end{array}$ & $\begin{array}{l}\text { Non-agriculteurs } \\
\text { originaires }\end{array}$ & $\begin{array}{l}\text { Agriculteurs } \\
\text { originaires }\end{array}$ & Total \\
\hline $\begin{array}{l}\text { Améliorer l'accès, établir des } \\
\text { résidences secondaires }\end{array}$ & 0 & 1 & 2 & 0 & 3 \\
\hline Se motiver, avoir plus de volonté & 0 & 0 & 2 & 2 & 4 \\
\hline Développer l'agriculture & 3 & 2 & 1 & 0 & 6 \\
\hline $\begin{array}{l}\text { Accorder des subventions pour la } \\
\text { restauration }\end{array}$ & 3 & 0 & 1 & 1 & 5 \\
\hline
\end{tabular}

Tab. 6: Mesures proposées pour l'entretien des terrasses 


\begin{tabular}{|l|l|l|l|l|l|}
\hline Problèmes mentionnés & $\begin{array}{l}\text { Néo- } \\
\text { ruraux }\end{array}$ & $\begin{array}{l}\text { Non-agriculteurs, } \\
\text { non-originaires }\end{array}$ & $\begin{array}{l}\text { Non-agriculteurs } \\
\text { originaires }\end{array}$ & $\begin{array}{l}\text { Agriculteurs } \\
\text { originaires }\end{array}$ & Total \\
\hline Emploi, économie & 0 & 0 & 5 & 1 & 6 \\
\hline Accès, route & 1 & 2 & 3 & 0 & 6 \\
\hline Dépeuplement & 1 & 0 & 0 & 2 & 3 \\
\hline Perte culture traditionnelle et agricole & 4 & 0 & 0 & 1 & 5 \\
\hline Dégradation de l'environnement & 4 & 1 & 0 & 0 & 5 \\
\hline Clientélisme, corruption & 1 & 2 & 0 & 0 & 3 \\
\hline
\end{tabular}

Tab. 7: Les principaux problèmes dans la vallée évoqués par les personnes interrogées

The main problems of the valley as mentioned by the persons interviewed

Die wichtigsten von den befragten Personen genannten Probleme des Tales

21 personnes interrogées. Cela montre que les personnes interrogées sont conscients et apprécient les particularités du paysage régional. Mais les terrasses, même si elles ont parfois été mentionnées comme élément typique du paysage, ne sont pas citées comme étant un aspect particulièrement attrayant. Peut-être que le mauvais état actuel des terrasses, comme le fait de ne plus être une ressource vitale, joue en défaveur de leur valorisation. Par ailleurs, le contact régulier avec les touristes, qui mettent surtout en valeur les caractéristiques générales du paysage méditerranéen et alpin, mais méconnaissent les terrasses, pourrait aussi influencer la perception de la population locale. L' «aspect sauvage» comme élément attrayant du paysage n'est cité que par 4 néo-ruraux.

Malgré la faible importance des terrasses dans la perception du paysage, la majorité des personnes interviewées estime important de les entretenir, mais tous ne partagent pas la même opinion sur la manière d'encourager cet entretien (tableau 6).

Les néo-ruraux interviewés se prononcent soit pour le développement de l'agriculture, soit pour les subventions directes à la restauration des terrasses. Quant aux personnes interrogées originaires de la vallée, ils sont plus nuancés sur les solutions qu'ils proposent. En plus de développer l'agriculture ou la restauration, certains pensent que l'abandon des terrasses est avant tout lié à un manque de volonté et de motivation des gens. D'autres proposent l'amélioration de l'accès et la vente de terrains comme résidences secondaires pour encourager la mise en valeur des terrasses. Cette dernière solution pourrait être liée aux personnes qui possèdent du terrain et ont un intérêt économique dans la spéculation foncière qui pourrait en résulter.

Le tableau 7 nous permet de mettre en relation ces réponses avec une vision plus générale du développement.
Les non-agriculteurs d'origine se préoccupent avant tout de l'emploi, de l'économie et des accès dans la vallée. Les néo-ruraux s'inquiètent surtout de la perte du savoir et de la culture traditionnelle et agricole, ainsi que de l'environnement. Les quatre personnes qui ont donné cette dernière réponse sont les mêmes qui citent l'aspect sauvage comme élément attrayant du paysage. Il est possible, sur cette base et d'après les autres questions, de distinguer deux groupes de néo-ruraux: un groupe «écologiste» qui a une opinion négative du tourisme, de l'Union Européenne (UE) et des pistes, et qui prend position en faveur de la protection fondamentale de l'environnement dans une approche biocentriste. L'autre groupe, plus modéré, valorise le savoir-faire agricole, artisanal et le bâti traditionnel dans la vallée, dans une approche anthropocentriste et sociale, proche de celle du développement durable.

Parmi les autres personnes interrogées, certains souhaitent une reprise agricole et un développement du tourisme dans la vallée, mais à condition de respecter l'environnement et la vie locale. D'autres, plutôt les personnes interrogées originaires de la vallée, souhaitent en priorité un développement économique avec la venue d'entreprises même industrielles, la construction de routes, le tourisme et l'installation de résidences secondaires ou de "rurbains» comme solution réaliste pour repeupler les campagnes et les entretenir. Ils considèrent parfois la démarche des néo-ruraux comme utopique. La différence entre villageois originaires ou non est cependant moins marquée qu'avec les néo-ruraux, et les opinions sont souvent plus nuancées par rapport au type de développement souhaité.

Le groupe des agriculteurs originaires a au contraire une attitude très positive face aux néo-ruraux. Même s'ils ne partagent pas leur idéologie, ils jugent leur présence comme une chance pour le renouveau de l'agriculture. 


\section{La succession: un processus réversible?}

D'après Di CASTRI (1981) le milieu méditerranéen est le résultat d'une co-évolution entre les peuples qui s'y sont développés et leur paysage culturel. Pour son équilibre, il dépend donc d'un dynamisme lié à l'activité humaine (NAVEH 1998). Dans le cas de la vallée de la Roya, un abandon total des terrasses à la forêt constituerait sans aucun doute une perte en diversité du paysage et d'espèces. C'est en particulier le cas en ce qui concerne l'abandon des prairies sèches.

Cependant, la lutte systématique contre les friches créerait un paysage statique avec forêts et cultures sans intermédiaires et diminuerait la diversité des écosystèmes. En théorie, il faudrait donc maintenir un équilibre dynamique où certaines zones cultivées comme les prairies sèches sont régulièrement abandonnées, alors que certaines friches sont régulièrement remises en culture.

Est-il possible de remettre des terrasses abandonnées en culture? Sur les prairies à Brachypodium pinnatum, une fauche régulière ne peut rétablir les prairies sèches que s'il en existe encore une extension suffisante pour permettre la recolonisation des espèces (Stampfli \& Zeiter 1999); or ce processus ne se fait qu'à long terme. Au stade arbustif, le débroussaillage est particulièrement difficile dans le maquis à cause des rejets de souches (DEBussche et al. 1996). Il en est de même pour le brûlis (Trabaud 1994). Un pâturage intensif pourrait empêcher la régénération des buissons, mais risque de détruire les murs des terrasses. Défricher la forêt et reconstruire les murs est possible, mais laborieux et coûteux. Certains habitants l'ont fait, mais à petite échelle $(<1 \mathrm{ha})$ et au prix d'un énorme travail.

Nous pouvons en conclure que la succession n'est pas réversible, ou ne l'est qu'au prix d'efforts considérables dès que les ligneux ont couvert la majeure partie du terrain. L'écroulement des murs des terrasses rend encore plus difficile la restauration de l'espace cultivable. En outre, comme l'ont montré Heiselmayer (1996) et Heiselmayer et al. (1995) dans la zone méditerranéenne en Grèce, et PIGNATTI (2003) concernant des types de végétation méditerranéenne anthropogénique, les écosystèmes restaurés sont nettement plus pauvres en espèces que les écosystèmes conservés. La conservation d'un écosystème est toujours plus efficace que sa restauration.

\section{Des scénarios pour l'avenir}

Dans la situation actuelle, les zones accessibles sont occupées par des résidences secondaires ou des villa- geois qui y entretiennent irrégulièrement les terrasses. Ailleurs, la forêt et les broussailles progressent sur les prés en friche. Les seules zones peu accessibles entretenues sont habitées par les néo-ruraux et de rares autres villageois, mais restent des cas isolés à l'avenir incertain.

Quatre différents scénarios pour l'avenir du paysage et des terrasses dans la vallée ont été identifiés en se basant sur les visions et les souhaits pour l'avenir exprimés par les habitants dans le sondage. Ces scénarios sont à comprendre comme des options de développement pour la vallée qui peuvent être combinées, et non comme des options unilatérales de ce qui pourrait réellement se passer à l'avenir.

\section{Scénario 1: Développement du tourisme périurbain}

La majorité des touristes se rendent régulièrement dans la vallée, et viennent souvent des villes proches ou de la côte. Ils souhaitent y établir un pied-à-terre, y achètent des terrains et y construisent des résidences secondaires. L'entretien des terrasses (murs et végétation) dépend de la volonté et de la disponibilité de chaque propriétaire. Comme les résidents secondaires exercent une activité professionnelle en ville ou sont des personnes âgées à la retraite, l'entretien des terrasses ne peut être que limité. Il ne se fait pas dans un but économique ou écologique, mais esthétique, avec éventuellement l'introduction d'espèces étrangères et de gazons artificiels, ou l'engraissement des prairies sèches, ce qui réduit leur biodiversité. Si les gens sont peu nombreux à installer leur résidence secondaire dans la vallée, ils n'occuperont que les zones les plus accessibles. En revanche, s'ils sont nombreux, les prix des terrains augmentent et découragent de nouveaux agriculteurs de s'établir dans la vallée.

\section{Scénario 2: Développement du tourisme rural}

A l'inverse du premier scénario, la majorité des touristes viennent irrégulièrement, ou de plus loin, et ne souhaitent pas acquérir de propriétés dans la vallée. Dans ce cas, l'effet d'une augmentation du flux touristique sur l'entretien du paysage dépend avant tout de la volonté des habitants de préserver ce qu'ils jugent attrayant autant pour eux que pour les touristes. Comme les terrasses ont été peu mentionnées comme un attrait du paysage par les personnes interrogées, il est peu probable que le tourisme encourage directement leur entretien. Elles seraient éventuellement préservées localement et à petite échelle dans le cadre d'un écomusée.

Une perspective plus intéressante pour le paysage traditionnel est le développement du tourisme «vert» ou familial («vacances à la ferme», gîte rural, visites culturelles, randonnée) qui pourrait procurer une source de revenu aux habitants. Dans ce cadre, l'entretien des 
terrasses pourrait être encouragé par des mesures de protection et un soutien spécifique en tant que patrimoine historique, mais aussi en favorisant le maintien d'activités agricoles locales à petite échelle, comme la production de "produits du terroir»-label écologique ou artisanal, destinés aux touristes.

\section{Scénario 3: Développement agricole}

De plus en plus de gens de l'extérieur s'installent dans la vallée pour y pratiquer l'agriculture, mais il est aussi possible que des habitants de la vallée reprennent, du moins à temps partiel, une activité agricole. Les effets sur le paysage dépendent des types d'exploitation agricole. Les oléiculteurs maintiennent naturellement les oliveraies, en entretenant ou non les murs des terrasses. L'élevage des ovins et caprins accélère l'écroulement des murs; les éleveurs de petit bétail ont alors tendance à transformer ceux-ci en talus (appelés «ribes» par la population locale). Pour l'élevage du gros bétail, on maintient les prairies sèches de fauche à Bromus erectus, mais elles pourraient être engraissées, ce qui provoquerait une diminution de la biodiversité.

Certaines zones sont déjà irréversiblement perdues pour l'exploitation agricole, impossible en l'absence de terrasses à cause de la pente. Le retour de l'agriculture se concentrerait sur les zones pas encore irréversiblement perdues, par exemple sur les adrets. Enfin, dans le cas d'une arrivée massive de néo-ruraux, des conflits avec la population originaire pourraient surgir.

Le type de développement des activités agricoles dépend fortement de la politique agricole et de protection de la nature. Des subventions à la production agricole risqueraient d'encourager l'usage d'engrais et de produits chimiques, donc de faire disparaître les prairies sèches. Le soutien à une agriculture écologique, avec pour but de maintenir une partie des prairies sèches et des oliveraies productives serait une solution acceptable et moins coûteuse qu'une protection et un entretien spécifique des terrasses comme patrimoine historique. Elle pourrait être mise en place dans le cadre du développement de la zone périphérique du Parc National du Mercantour, dont les communes de Breil-sur-Roya, Saorge et Fontan font partie.

\section{Scénario 4: Développement économique}

Des entreprises ou des unités d'administration décentralisées s'installent et embauchent dans la vallée, ce qui permet à sa population d'y trouver du travail et d'y rester. Le développement d'entreprises industrielles est peu probable, vu les contraintes d'accès liées à la topographie, mais des entreprises liées aux services pourraient trouver avantageux les prix des terrains dans la vallée par rapport à la côte. Comme avec les résidences secondaires, les gens entretiennent leurs terrains durant leur temps libre. Mais ils peuvent mieux le faire car ils vivent sur place; ils peuvent continuer de cultiver des jardins potagers, faire de l'huile d'olive et des conserves pour leur propre consommation, comme certains villageois originaires le font encore par tradition. La condition est que leur travail leur laisse du temps libre, ce qui dépend de la politique sociale des entreprises comme de l'Etat. La présence de certaines entreprises pourrait cependant diminuer l'attrait du paysage pour les touristes et les néo-ruraux, et favoriser l'abandon de certains secteurs. Ce scénario, bien que souhaité par une partie de la population, reste cependant peu probable, à moins de songer à des entreprises liées au tourisme, ou à l'élaboration semiartisanale de produits du terroir, comme par exemple l'huile d'olive.

Ces scénarios montrent que plusieurs options de développement pourraient encourager l'entretien des terrasses, et doivent être combinées. A cause de la proximité de la Côte d'Azur, le plus grand potentiel de développement de la vallée reste le tourisme. Le développement de l'agriculture ou l'arrivée d'entreprises reste peu probable, à moins que ces activités ne soient liées au tourisme. C'est justement pour cela qu'un tourisme culturel et écologique doit être encouragé, vu qu'il permet de stimuler des activités annexes comme la vente de produits du terroir, bruts ou élaborés, et par conséquent l'entretien des terrasses.

\section{Conclusions}

La majeure partie des terrasses de culture de la moyenne Roya est à l'abandon. Dans presque tous les cas, la prairie à Brachypodium pinnatum qui s'y installe laisse place à une végétation arbustive, puis à long terme à la forêt, proche de la végétation naturelle potentielle. A cause de l'écroulement des murs comme de la régénération des ligneux après une éventuelle coupe ou un brûlis, ce processus provoque une perte irréversible de terres potentiellement cultivables à une échelle économique et temporelle réaliste. Dans les circonstances actuelles, la plus grande partie des terrasses de la vallée est donc appelée à disparaître, ou a déjà disparu.

Cette évolution ne correspond pas aux attentes des personnes interrogées de la vallée, qui souhaitent entretenir le paysage, mais ne peuvent en faire une priorité. Autant les villageois originaires que les néoruraux adhèrent à une forme de développement rural mais avec des conceptions différentes. En souhaitant la venue d'entreprises, un développement industriel et plus d'infrastructures, certains villageois originaires cherchent avant tout à échapper à la marginalité qui leur est attribuée en tant que ruraux. Les néo-ruraux, qui souhaitent un renouveau de l'agriculture et des 
activités traditionnelles, ont tendance à valoriser, voire idéaliser un espace vu comme une richesse parce qu'il est choisi et non vécu comme une contrainte (DESMICHEL 2000). Ces divergences d'opinion ont pour conséquence, au moins jusqu'à maintenant, l'absence d'un projet commun de développement.

Pour la conservation des terrasses ainsi que de la diversité des espèces et des écosystèmes, la priorité est de maintenir un espace ouvert et cultivé, et donc de lutter contre la progression des friches. Mais en second lieu, il faut éviter des limites trop nettes entre cultures et forêts en créant des zones de transition (écotones), comme des haies ou des lisières de forêt, que l'on coupe de temps en temps, afin de créer une dynamique qui favorise une biodiversité élevée (voir par exemple KrüsI et al. 1997). Encore faut-il savoir quel type de terrasses devraient être entretenues, s'il s'agit des terrasses de cultures céréalières et maraîchères - qui ont actuellement complètement disparu -, des prairies sèches, ou des oliveraies. Si l'on donne la priorité à la conservation des espèces, les prairies sèches avec leurs orchidées, donc l'élevage et, par exemple, la production de fromage artisanal, sont à encourager. Dans ce cas, l'effet sur les terrasses n'est pas forcément positif, à cause de l'érosion par le bétail. Si l'on accorde la priorité à la conservation des terrasses, des zones de cultures maraîchères pourraient être rétablies, en plus des oliveraies, pour fabriquer des "produits du terroir», frais ou en conserve, destinés aux touristes. Pour conserver autant les terrasses que la biodiversité, un équilibre doit donc être trouvé entre la conservation de différents types d'activités sur les terrasses.

Une restauration directe des terrasses dans l'unique but de les conserver serait très coûteuse s'il fallait la réaliser à grande échelle. Un tourisme lié aux résidences secondaires ne laisserait que peu de temps aux habitants pour entretenir leurs terrains, mais la présence d'un tourisme culturel et écologique pourrait stimuler certaines activités agricoles liées aux terrasses. Il est cependant illusoire de croire que la seule loi du marché, même par le biais du tourisme, puisse stimuler l'entretien des terrasses. A cause de leur faible productivité, il est impossible que des cultures maraîchères, céréalières ou l'exploitation des prairies sèches puisse perdurer dans les conditions économiques actuelles. Par conséquent, il est impératif d'implanter une politique agricole favorable aux produits locaux, par exemple au moyen de subventions écologiques couplées à des taxes sur les produits conventionnels et importés, de labels écologiques ou d'Appellation d'Origine Contrôlée (AOC). D'une part, il s'agit de protéger les espèces rares des prairies sèches en encourageant l'élevage à petite échelle et la production de fromage artisanal. Cela implique une révision des normes d'hygiène draconiennes de l'UE qui, pour le moment, ne permettent pas ce genre d'activités. D'autre part, il s'agit de protéger le patrimoine culturel des terrasses en encourageant l'oléiculture et d'autres produits du terroir destinés avant tout à la vente aux touristes.

Finalement, la mise en place de mesures pour encourager l'entretien des terrasses ne pourra être efficace que dans le cadre d'une bonne coordination d'activités et d'une bonne entente entre les différents groupes sociaux de la vallée. Il faudrait pour cela renforcer la cohésion sociale en organisant par exemple des ateliers de réflexion et des groupes de discussion.

\section{Références}

AlbreCHT, M.(2001): Sekundäre Sukzessionsprozesse auf nicht mehr bewirtschafteten Terrassenkulturflächen bei Saorge (Alpes-Maritimes). - Unveröffentlichte Diplomarbeit, Universität Zürich, Geographisches Institut.

Barbero, M., Bono, P.-G., Ozenda, P. \& G.-P. Mondino (1973): Carte écologique des Alpes au 1/100 000. NiceMenton et Viève-Cuneo, Coupe des Alpes maritimes et ligures. - In: Documents de cartographie écologique 12: 49-76.

Blanchemanche, P. (1990): Bâtisseurs de paysages. Terrassement, épierrement et petite hydraulique agricoles en Europe XVIIe - XIXe siècles. - Paris: Maison des Sciences de l'Homme.

Boillat, S. (2002): La succession végétale sur les anciennes cultures en terrasses de la vallée de la Roya (Alpes-Maritimes) et sa perception par la population locale. - Travail de diplôme, Institut Géobotanique, EPF Zürich (non publié).

Botron, C. (1996): Histoire de Breil et des Breillois. Breil-sur-Roya: Les Editions du Cabri.

Braun-Blanquet, J. (1964): Pflanzensoziologie. - Wien: Springer.

Cevasco, J.-M. \& Association des amis de la BenDOLA (2001): Inventaire floristique et faunistique de la vallée de la Bendola. - Saorge (non publié).

Debussche, M., Escarre, J., Lepart, J., Houssard, C. \& S. LAVOREL (1996): Changes in Mediterranean plant succession, old-fields revisited. - In: Journal of Vegetation Science 7: 519-526.

Desmichel, P. (2000): Réalité économique et perception sociale du tourisme en milieu rural fragile - Analyse à travers l'exemple de trois territoires pyrénéens. - In: Revue de Géographie Alpine 88: 51-63.

Di CASTRI, F. (1981): Mediterranean-type shrublands of the world. - In: Ecosystems of the world 11: 1-52 EllenberG, H. (1996): Vegetation Mitteleuropas mit den Alpen. - 5ème éd., Stuttgart: Ulmer.

Fournier, P. (1990): Les quatre flores de France. Paris: Editions Lechevalier.

Flick, U. (2000): Qualitative Forschung. Theorie, Methoden, Anwendung in Psychologie und Sozialwissenschaften. - Hamburg: Rowohlt. 
Frapa, P., Ambroise, R. \& S. Giorgis (1989): Paysages de terrasses. - Aix-en-Provence: Edisud.

GlavAC, V. (1996): Vegetationsökologie. Grundfragen, Aufgaben, Methoden. - Jena: Gustav Fischer.

Heiselmayer, P. (1996): Menschlich bedingte Vegetationsveränderungen auf den Kykladen (Ägäis). - In: Archiv für Natur- und Landschaftsschutz 35: 161-174.

Heiselmayer, P., Grabner, S. \& B. Giehser (1995): Vegetation und Vegetationsentwicklung der Phrygana in Mykonos und Tinos. - In: Salzburger Geographische Arbeiten 29: 233-266.

KöHLER, B. (2001): Mechanisms and extent of vegetation changes in differently managed limestone grasslands. - Dissertation, Geobotanisches Institut, ETH Zürich.

KRÜSI, B.O., SchÜTZ, M. \& S. Tidow (1997):Waldränder in der Schweiz. Ökologischer Zustand, botanische Vielfalt und Aufwertungspotential. - In: Schweizer Wald 97, 4: 5-26.

LAmNeK, S. (1995): Qualitative Sozialforschung, Tome 2: Methoden und Techniken. - 3ème éd., Weinheim: Beltz-Psychologie Verlags Union.

LeuterT, A. (1983): Einfluss der Feldmaus Microtus arvalis (Pall.) auf die floristische Zusammensetzung von Wiesen-Oekosystemen. - In: Veröffentlichungen des Geobotanischen Instituts ETH, Stiftung Rübel, Zürich 79: 1-126.

NAveh, Z. (1998): From Biodiversity to Ecodiversity, Holistic Conservation of the Biological and Cultural Diversity of Mediterranean Landscapes. - In: Ecological Studies 136: 23-53.

Ne'eman, G. \& I. Izhaki (1996): Colonization in an abandoned East-Mediterranean vineyard. - In: Journal of Vegetation Science 7: 465-472.

Ozenda, P. (1981): Végétation des Alpes sud-occidentales. - Paris: CNRS Editions.

Pignatti, S. (2003): The mediterranean ecosystem. - In: Bocconea 16: 29-40.

RiCHTER, M. (1989): Untersuchungen zur Vegetationsentwicklung und zum Standortwandel auf mediterranen Rebbrachen. - In: Braun-Blanquetia 4: 1-196.

SchmidT, W. (1985): Mahd ohne Düngung. Vegetationskundliche und ökologische Ergebnisse aus Dauerflächenuntersuchungen zur Pflege von Brachflächen. - In: Münstersche Geographische Arbeiten 20: 81-99.

SchmidT, W. (1993): Sukzession und Sukzessionslenkung auf Brachäckern. Neue Ergebnisse aus einem Dauerflächenversuch. - In: Scripta Geobotanica 20: 65-104.

Stampfli, A. \& M. Zeiter (2001): Species responses to climatic variation and land-use change in grasslands of southern Switzerland. - In: BURGA, C.A. \& A. KRATOCHWIL (eds): Biomonitoring: General and applied aspects on regional and global scales. - Tasks for Vegetation Science 35: 107-124, Dordrecht, Boston, London: Kluwer.
TATONI, T. \& P. Roche (1994): Comparison of old-field and forest revegetation dynamics in Provence. - In: Journal of Vegetation Science 5: 295-302.

TRABaud, L. (1994): Postfire plant community dynamics in the Mediterranean Basin. - In: Ecological Studies 107: 1-15.

\section{Résumé: La succession végétale sur les cultures en terrasses de la Vallée de la Roya (Alpes-Maritimes, France) et sa perception par la population locale}

La présente étude met en relation l'état écologique des cultures en terrasses de la moyenne Vallée de la Roya avec les valeurs socioculturelles qui y sont liées.

Quatorze types de végétation ont été identifiés et ordonnés dans un modèle de succession. Les terrasses encore entretenues sont des oliveraies ou des prairies sèches. A l'arrêt de la fauche, une prairie à Brachypodium pinnatum s'installe, suivie par un stade arbustif qui évolue ensuite en forêt. Les proportions de surface des différents types de végétation montrent que les zones plus peuplées et mieux accessibles possèdent la plus grande surface de terrasses entretenues.

Un sondage parmi 21 habitants a permis de cerner leur prise de position face à l'abandon des terres, ainsi que leur vision d'avenir pour la vallée. Le spectre des opinions va de celle des néo-ruraux, qui considèrent le renouveau de l'agriculture traditionnelle comme un moteur de développement, à celle de villageois autochtones qui voient dans le développement de l'industrie et du tourisme les seules perspectives réalistes d'avenir.

A partir de ces résultats, quatre scénarios sur l'avenir de la vallée et ses conséquences sur l'entretien des terrasses ont été élaborés et discutés.

Summary: Vegetational succession on abandoned terraced land in the Vallée de la Roya (Alpes-Maritimes, France) and its perception by local people

The paper aims to show the connection between the ecological state of terraced land in the middle section of Roya valley and the socio-cultural values linked thereto.

Fourteen vegetation types were identified and grouped in a succession model. At present, maintained terraces are characterised by olive groves or limestone grasslands. If left uncut, the terraces are initially colonised by Brachypodium pinnatum grassland, to be followed by scrubland (maquis), and finally forest. Spatial mapping of different vegetation types show that the largest percentage of maintained terraces is to be found in regions with the highest population density and easy accessibility to the terraces. 
Interviews with 21 inhabitants allowed for information to be collected on local opinions concerning land abandonment and future prospects of the valley. The opinion spectrum ranged from neo-rurals, who considered the rediscovering of traditional agriculture as a development perspective, to local villagers who saw industry and tourism development as the only realistic prospect for the future.

Based on these results, four scenarios for the valley's future were elaborated and discussed, with particular attention being paid to the consequences of each scenario for the maintenance of terraced land.

\section{Zusammenfassung: Vegetations-Sukzessionen auf Terrassenkulturen des Vallée de la Roya (Alpes- Maritimes, Frankreich) und ihre Wahrnehmung durch die lokale Bevölkerung}

Diese Studie untersucht im mittleren Teil des Vallée de la Roya Vegetations-Sukzessionen auf terrassiertem Kulturland und dessen soziokulturelle Bedeutung.

Es wurden 14 Vegetationstypen unterschieden und in einem Sukzessionsmodell gruppiert. Auf den heute noch bewirtschafteten Terrassen hat es meist Olivenhaine oder Trockenwiesen. Bei fehlender Mahd stellt sich eine Brachypodium pinnatum-Wiese ein, gefolgt von einem Strauch-Stadium, das in Wald übergeht. Bezogen auf die Anteile der verschiedenen Vegetationstypen weisen die am dichtesten besiedelten und am besten zugänglichen Zonen den grössten Anteil an gepflegten Terrassenkulturen auf.

Eine Befragung von 21 Bewohnern lieferte Informationen zur Aufgabe des Kulturlandes und zu den Zukunftsperspektiven ihres Tales. Das Spektrum der Ansichten reicht von den «Neu-Bauern» (Aussteiger, «néo-ruraux»), welche die traditionelle Landwirtschaft im Sinne einer Entwicklungsperspektive beleben wollen, bis zu den einheimischen Dorfbewohnern, die in der Förderung von Industrie und Tourismus das einzige realistische Zukunfts-Szenario sehen.

Beruhend auf diesen Untersuchungsergebnissen wurden vier Zukunfts-Szenarien für das Tal und deren Konsequenzen für den Unterhalt des terrassierten Geländes erarbeitet und diskutiert.

\section{Orientations didactiques}

- Quelles furent les étapes principales du développement de la Vallée de la Roya au courant du XXe siècle?

- Quelles sont les causes de l'abandon des terrasses de cultures dans les Alpes-Maritimes ?

- Quels sont les signes de dégradation des terrasses de cultures après l'abandon?

- Quelle succession de stades végétaux peut-on observer sur les terrasses couvertes de prairies? Quels sont les facteurs marquants de cette succession? Que peut-on dire de la diversité des plantes, dès lors que l'on compare des systèmes écologiques conservés avec des systèmes restaurés?

- Comment peut-on expliquer l'état différencié des terrasses-murets?

- Quels scénarios peut-on considérer pour l'avenir des terrasses de cultures abandonnées?

- Quels sont les éléments du paysage qui sont considérés par les interviewés comme particulièrement attractifs?

- Quelles sont les orientations que les quatre groupes différenciés de la population interviewés souhaiteraient donner au développement de la vallée? Quels sont les avantages et les inconvénients?

Dipl. Natw. ETH Sébastien Boillat, Geobotanical Institute ETH-Z, Gladbachstrasse 114, CH-8044 Zurich. e-mail:agruco@entelnet.bo

Prof. Dr. Conradin A. Burga, Department of Geography, University of Zurich-Irchel, Winterthurerstrasse 190, CH-8057 Zurich.

e-mail: cburga@geo.unizh.ch

Prof. Dr. Andreas Gigon, Geobotanical Institute ETH-Z, Gladbachstrasse 114, CH-8044 Zurich.

e-mail: andreas.gigon@env.ethz.ch

PD Dr. Norman Backhaus, Department of Geography, University of Zurich-Irchel, Winterthurerstrasse 190, CH-8057 Zurich.

e-mail:backhaus@geo.unizh.ch

\section{Manuskripteingang/received/manuscrit entré le}

1.12.2003

Annahme zum Druck/accepted for publication/accepté pour l'impression: 24.5.2004 